\title{
Enterocytozoon bieneusi in donkeys from Xinjiang, China: prevalence, molecular characterization and the assessment of zoonotic risk
}

\author{
Aiyun Zhao ${ }^{1}$, Ying Zhang ${ }^{1}$, Wen Wang ${ }^{1}$, Bo Jing ${ }^{1}$, Jinming Xing ${ }^{1}$, Dayong Tao ${ }^{1}$, Wei Zhao ${ }^{2^{*}}$ and Meng Qi ${ }^{\text {* }^{*}}$
}

\begin{abstract}
Background: Enterocytozoon bieneusi, a zoonotic pathogen, has the potential to infect both immunocompromised and immunocompetent humans. It is found in large number of animals; however, not much is known regarding its prevalence in equine animals, particularly donkeys. This is the first molecular epidemiological evaluation of $E$. bieneusi in 178 free-ranging donkeys from five countrysides; and 502 farmed donkeys from 18 farms in 12 cities of Xinjiang, China by Nested PCR.

Results: E. bieneusi was detected in 2.5\% (17/680) donkeys, with 2.6\% (13/502) in farmed and 2.2\% (4/178) in freeranging ones. Sequence analysis identified eight ITS genotypes, all belonging to zoonotic Groups 1 or 2, including six known genotypes: horse1 $(n=5), \mathrm{D}(n=3), \operatorname{NCD}-2(n=3), \operatorname{BEB} 6(n=2), \operatorname{BEB} 4(n=1)$, and NIAI $(n=1)$; and two new genotypes: XJD1 $(n=1)$ and XJD2 $(n=1)$.
\end{abstract}

Conclusions: This is the first report confirming the presence of E. bieneusi in donkeys in Xinjiang, China, and indicates the possibility of zoonotic transmission of this pathogenic parasite.

Keywords: Enterocytozoon bieneusi, Zoonotic, ITS region, Genotype, Donkey

\section{Background}

Microsporidia are obligate, spore-forming, intracellular pathogens, comprising more than 200 genera and 1500 species that are infectious to a numerous animals (both vertebrates and invertebrates) [1]. Of these, 17 species are known to be infectious to humans. Amongst these, Enterocytozoon bieneusi (E. bieneusi) is prevalent in humans and is known to cause life-threatening infections in people with a weak immune system [2]. Due to the global increase in the number of cases of E. bieneusi infection, it has been classified as an emerging infectious

\footnotetext{
*Correspondence: hayidazhaowei@163.com; qimengdz@163.com

${ }^{2}$ Department of Parasitology, Wenzhou Medical University, Wenzhou 325035, Zhejiang Province, China

${ }^{1}$ College of Animal Science, Tarim University, Tarim Road 1487, Alar 843300, Xinjiang, China
}

\section{$\triangle B M C$}

C C The Author(s). 2020 Open Access This article is licensed under a Creative Commons Attribution 4.0 International License, which permits use, sharing, adaptation, distribution and reproduction in any medium or format, as long as you give appropriate credit to the original author(s) and the source, provide a link to the Creative Commons licence, and indicate if changes were made. The images or other third party material in this article are included in the article's Creative Commons licence, unless indicated otherwise in a credit line to the material. If material is not included in the article's Creative Commons licence and your intended use is not permitted by statutory regulation or exceeds the permitted use, you will need to obtain permission directly from the copyright holder. To view a copy of this licence, visit http://creativecommons.org/licenses/by/4.0/ The Creative Commons Public Domain Dedication waiver (http://creativecommons.org/publicdomain/zero/1.0/) applies to the data made available in this article, unless otherwise stated in a credit line to the data.

disease [3]. This parasite has also been identified in animals as well as water supplies, supporting the possibility of zoonotic infections with water contact acting as the transmission vehicle for the parasite [4-6].

Genotyping studies on the polymorphism of the internal transcribed spacer (ITS) region have revealed genetic mutations in E. bieneusi isolated from both domestic and wild animals, humans, and surface water [1, 2, 4, 7]. Currently, phylogenetic analysis has clustered the 470 known genotypes of E. bieneusi into 11 genetically-isolated groups [3]. Groups 1 and 2 include most humans and zoonotic genotypes, and thus, are important for public health, while Groups 3 to 11 constitute those genotypes that have adapted to specific hosts or wastewater, suggesting a reduced risk to public health [3]. Epidemiological studies 
focusing on genotyping the $E$. bieneusi extracts from those animals that have a higher probability of human contact would expand our current knowledge on the (a) prevalence of human microsporidiosis and (b) the zoonotic transmission of E. bieneusi.

There is a scarcity of data on the prevalence of $E$. bieneusi in equine animals, particularly donkeys [814]. Based on historical data, for the last 5000 years in China, donkeys, with a population exceeding 11 million, have been used for carrying heavy loads or for draught work in transportation/agriculture. They are raised for milk, meat, and Ejiao, a traditional Chinese medicine. Xinjiang Uygur Autonomous Region (hereafter referred to as Xinjiang) is the birthplace of China's donkeys, and nearly a million donkeys living here. However, there is a scarcity of data on the prevalence of E. bieneusi in those animals from Xinjiang [13].. Herein, we evaluated the possibility of zoonotic transmission of $E$. bieneusi along with its prevalence in free-ranging and scale-farmed donkeys in the Xinjiang in China.

\section{Results}

Prevalence of $E$. bieneusi in donkeys

Six hundred and eighty fecal samples from donkeys were analyzed using nested-PCR to study the prevalence of $E$. bieneusi. We found E. bieneusi in 2.5\% (17/680) of donkeys, with $2.6 \%$ (13/502) in farmed animals and 2.2\% (4/ $178)$ in free-ranging animals. For the farmed donkeys, Yopurga $(7 / 103 ; 6.8 \%)$ showed the highest infection rate of $E$. bieneusi, followed by Khorgas (1/20; 5.0\%), Karakax $(4 / 88 ; 4.5 \%)$, and Bohu $(1 / 79 ; 1.3 \%)$. Other sampled farms showed no cases of $E$. bieneusi infection. For freeranging donkeys, $E$. bieneusi was identified at two collection sites with infection rates of 5.9\% (2/34) in Zepu and $3.1 \%(2 / 64)$ in Yecheng. No E. bieneusi infection was detected at the other three sites (Table 1). No statistically significant difference in the infection rates was observed between young and adult donkeys, which had a prevalence of E. bieneusi as 2.4 and $2.8 \%$ respectively, $(p>$ $0.05)$. The study did not include young free-ranging donkeys (Table 2).

\section{E. bieneusi genotypes in donkeys}

We identified eight $E$. bieneusi genotypes in this study, which included six known genotypes: horse1 $(n=5)$, D $(n=3)$, NCD-2 $(n=3)$, BEB6 $(n=2)$, NIAI $(n=1)$, and BEB4 $(n=1)$, and two new genotypes: XJD1 $(n=1)$ and XJD2 $(n=1)$. Both new genotypes: XJD1 (MN174117) and XJD2 (MN174120) were found to be closely associated with genotypes BEB4 and $\mathrm{O}$, with one and two nucleotide variations, respectively. Amongst the genotypes, horse1, BEB4, XJD2, and NCD-2 were found in farmed donkeys; XJD1 and NIA1 were found in free-ranging
Table 1 Prevalence and genotype distribution of Enterocytozoon bieneusi in in donkeys in Xinjiang

\begin{tabular}{|c|c|c|c|}
\hline $\begin{array}{l}\text { Feeding } \\
\text { pattern }\end{array}$ & $\begin{array}{l}\text { Collection } \\
\text { site }\end{array}$ & $\begin{array}{l}\text { No. Positive/ } \\
\text { No. Samples } \\
\text { (\%) }\end{array}$ & $\begin{array}{l}\text { E. bieneusi } \\
\text { genotype (n) }\end{array}$ \\
\hline \multirow[t]{12}{*}{ Farmed } & Alaer & $0 / 54$ & - \\
\hline & Barkol & $0 / 11$ & - \\
\hline & Bohu & $1 / 79(1.3)$ & BEB6 (1) \\
\hline & Gongliu & $0 / 21$ & - \\
\hline & Huocheng & $0 / 20$ & - \\
\hline & Khorgas & $1 / 20(5.0)$ & BEB4 (1) \\
\hline & Karakax & $4 / 88(4.5)$ & NCD-2 (3), XJD2 (1) \\
\hline & Pishan & $0 / 41$ & - \\
\hline & Qitai & $0 / 16$ & - \\
\hline & Turpan & $0 / 17$ & - \\
\hline & Yuli & $0 / 32$ & - \\
\hline & Yopurga & $7 / 103(6.8)$ & horse1 (5), D (2) \\
\hline Subtotal & & 13/502 (2.6) & $\begin{array}{l}\text { horse1 (5, NCD-2 (3), D (2), } \\
\text { XJD2 (1), BEB4 (1), BEB6 (1) }\end{array}$ \\
\hline \multirow[t]{5}{*}{ Domestics } & Akqi & $0 / 11$ & - \\
\hline & Barkol & $0 / 21$ & - \\
\hline & Pishan & $0 / 48$ & - \\
\hline & Yecheng & $2 / 64(3.1)$ & BEB6 (1), NIA1 (1) \\
\hline & Zepu & 2/34 (5.9) & D (1), XJD1 (1) \\
\hline Subtotal & & $4 / 178(2.2)$ & $\mathrm{D}(1), \mathrm{XJD} 1$ (1), BEB6 (1), NIA1 (1) \\
\hline Total & & $17 / 680(2.5)$ & $\begin{array}{l}\text { horse1 (5), D (3), NCD-2 (3), BEB6 } \\
\text { (2), BEB4 (1), XJD1 (1), XJD2 (1), } \\
\text { NIA1 (1) }\end{array}$ \\
\hline
\end{tabular}

animals; genotypes BEB6 and D were identified in both feeding groups.

\section{Phylogenetic analysis}

Phylogenetic analysis showed that group 1 genotypes were horse1, D, NIA1, NCD-2, and XJD2; and group 2 genotypes were BEB6, BEB4, and XJD1 (Fig. 1).

Table 2 Prevalence and distribution of Enterocytozoon bieneusi genotypes according to feeding pattern and age in donkeys in Xinjiang

\begin{tabular}{lll}
\hline $\begin{array}{l}\text { Feeding } \\
\text { pattern/ Age }\end{array}$ & $\begin{array}{l}\text { No. Positive/No. } \\
\text { Samples (\%) }\end{array}$ & E. bieneusi genotype (n) \\
\hline free-ranging & $4 / 178(2.2)$ & BEB6 (1), D (1), XJD1 (1), NIA1 (1) \\
Scale farming & $13 / 502(2.6)$ & $\begin{array}{l}\text { BEB4 (1), BEB6 (1), D (2), horse1 (5), } \\
\text { XJD2 (1), NCD-2 (3) }\end{array}$ \\
$<1$ years & $7 / 296(2.4)$ & $\begin{array}{l}\text { D (2), XJD2 (1), horse1 (1), NCD-2 } \\
(3)\end{array}$ \\
$>$ 1 years & $9 / 323(2.8)$ & BEB6 (2), D (1), XJD1 (1), horse1 (4), \\
Unclear & $1 / 61(1.6)$ & BIA1 (1) \\
\hline
\end{tabular}




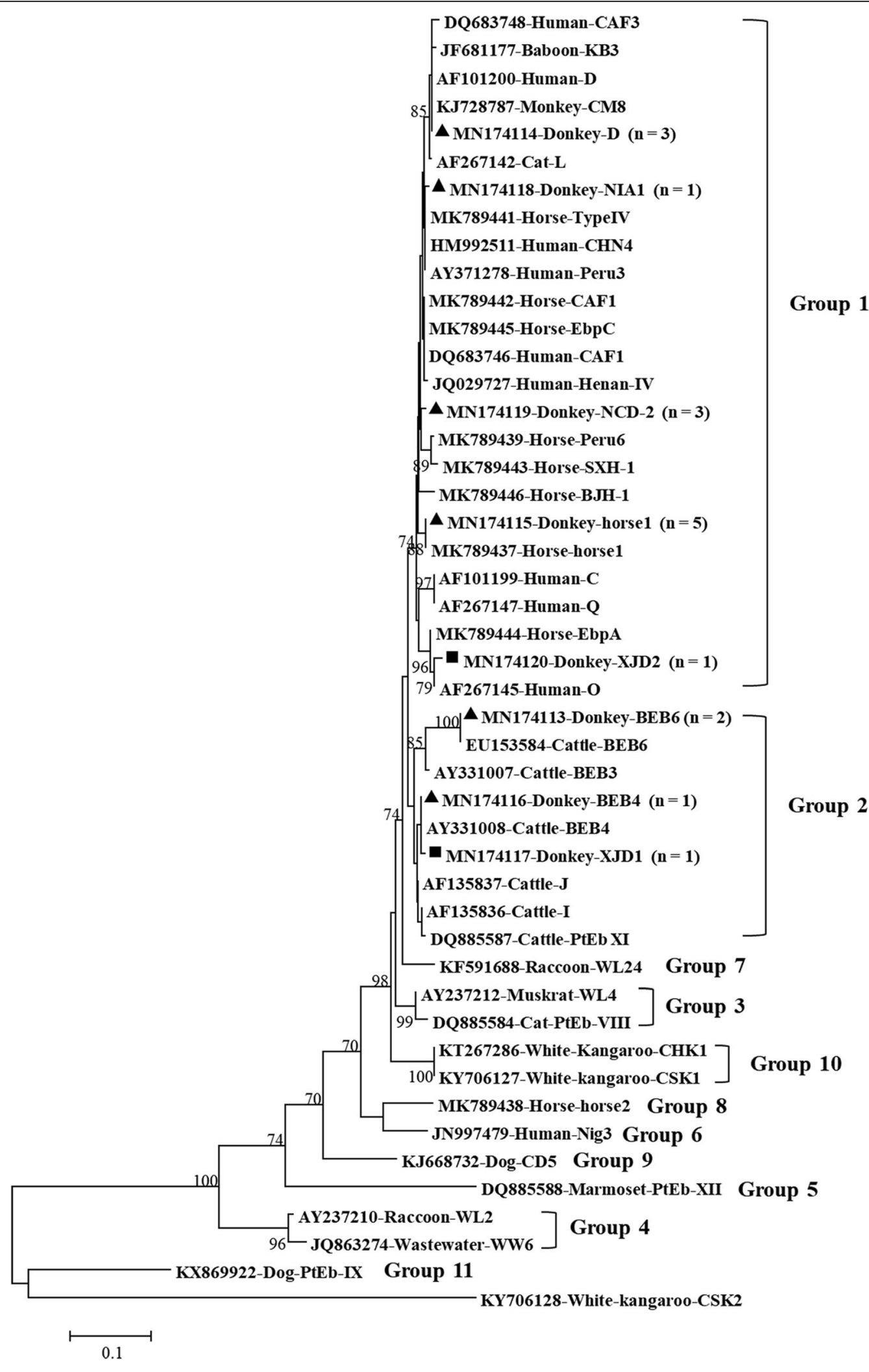

Fig. 1 (See legend on next page.) 
(See figure on previous page.)

Fig. 1 ITS sequence-based phylogenetic tree. Phylogenetic relationship between the known (GenBank) and identified (this study) E. bieneusi genotypes were identified through an NJ analysis based on the Kimura two-parameter model. Sequence detection was performed based on its host origin, accession number, and the designated genotype. The branches show the percent bootstrapping values from 1000 replicates. Outgroup classification comprised of the E. bieneusi genotype CSK2 (KY706128) from white kangaroo. The filled triangles and squares indicate known and novel genotypes, respectively

\section{Discussion}

Previous studies have reported that in equine animals, the rates of infection of E. bieneusi fall in the range of 1.6 to $30.9 \%$. However, there is a scarcity of data on the prevalence of E. bieneusi in donkeys (Table 3) [8-14]. Globally, only two studies, based in Algeria and China, have identified $E$. bieneusi in donkeys with infection rates of $1.6 \%$ (2/ $124)$ and $5.3 \%(16 / 301)$, respectively $[8,13]$. Here, we reported the prevalence of E. bieneusi to be $2.5 \%$, which was higher than 1.6\%, which was reported in Algeria and lower than the $5.3 \%$ previously reported in China $[8,13]$. These differences could be attributed to geographical factors, variations in feeding density, sample sizes, and differential management systems. In this study, the prevalence of $E$. bieneusi in farmed and free-ranging donkeys were $2.6 \%$ $(13 / 502)$ and $2.2 \%(4 / 178)$, respectively and no statistical differences in infection rates were observed between them.

Table 3 Prevalence and genotype distribution of Enterocytozoon bieneusi in equines worldwide

\begin{tabular}{|c|c|c|c|c|}
\hline Host & Country & $\begin{array}{l}\text { Positive } \\
\text { no. / total } \\
\text { no. (\%) }\end{array}$ & Genotype (no.) & Reference \\
\hline \multirow[t]{5}{*}{ Horse } & Algeria & $\begin{array}{l}6.8(15 / \\
219)\end{array}$ & $\begin{array}{l}\text { horse1 (6), CZ3 (2), D (1), } \\
\text { horse2 (1), Unknown (5) }\end{array}$ & {$[8]$} \\
\hline & Colombia & $\begin{array}{l}10.8(21 / \\
195)\end{array}$ & $\begin{array}{l}\text { horse1 (13), D (4), horse2 } \\
\text { (4) }\end{array}$ & [9] \\
\hline & China & $\begin{array}{l}22.5(75 / \\
333)\end{array}$ & $\begin{array}{l}\text { SCO2 (16), horse1 (13), D } \\
\text { (1), SCH1(1), SCH3 (1), } \\
\text { horse2 (39), YNH1 (1), } \\
\text { YNH2 (1), SCH4 (1) }\end{array}$ & [10] \\
\hline & China & $\begin{array}{l}30.9(81 / \\
262)\end{array}$ & $\begin{array}{l}\text { EbpC (21), EpbA (20), CS-4 } \\
\text { (4), horse1 (4), O (4), G (3), } \\
\text { PigEBITS4 (2), CM8 (1), CS-1 } \\
\text { (1), CS-4 (1), D (1), ESH-01 } \\
\text { (1), Peru8 (1), XJH3 (1), } \\
\text { BEB6 (9), CM7 (2), horse2 } \\
\text { (2), XJH1 (2), XJH4 (1) }\end{array}$ & [11] \\
\hline & Czech R & $\begin{array}{l}17.5(66 / \\
377)\end{array}$ & $\begin{array}{l}\text { D (34), horse1 (7), G (3), } \\
\text { EpbA (2), horse11 (2), } \\
\text { horse4 (1), horse10(1), } \\
\text { WL15 (1), horse2 (8), } \\
\text { horse3 (2) }\end{array}$ & [12] \\
\hline \multirow[t]{2}{*}{ Donkey } & Algeria & $\begin{array}{l}1.6(2 / \\
124)\end{array}$ & Unknown (2) & [8] \\
\hline & China & $\begin{array}{l}5.3(16 / \\
301)\end{array}$ & $\begin{array}{l}\mathrm{D}(4), \mathrm{NCD}-1 \text { (1), NCD-2 (1), } \\
\text { J (10) }\end{array}$ & [13] \\
\hline Mustang & USA & $8.3(7 / 84)$ & horse1 (7) & [12] \\
\hline Zebra & China & $20.0(1 / 5)$ & $J(1)$ & [14] \\
\hline
\end{tabular}

However, in fact, the present study did not include any young free-ranging donkeys, only young farmed donkeys, which might have skewed the infection rate findings.

Previous epidemiological studies have reported the existence of 17 genotypes of $E$. bieneusi in equine animals with only four genotypes (D, NCD-1, NCD-2, and J) in donkeys (Table 3) [8-14]. Among these genotypes, $\mathrm{D}$ and $\mathrm{J}$ are the most common [8, 13]. Genotype D is a zoonotic genotype and has been reported in more than 20 countries, and isolated from more than 25 animal species, apart from water samples $[3,15,16]$. Genotype J was initially considered to be cattle-based as it was discovered in cattle from China, Argentina, Korea, Germany, the USA, and Portugal [3, 17]. However, recently, this genotype has been discovered in pigs, human, and non-human primates (NHPs) in China [18-20], as well as in other animals, including alpaca, sheep, goat, yak, deer, zebra, bear, and meerkats [3, 21]. The genotype has also been reported in water samples from China [22], in deer from Australia and the USA [23], and in birds from Iran [24]. Until now, genotypes NCD-1 and NCD-2 have only been identified in donkeys [13].

Here, we identified four previously known genotypes (horse1, BEB6, BEB4, and NIAI) and two new genotypes (XJD1 and XJD2), apart from the genotypes D and NCD-2, in donkeys. Genotype horse1 showed good adaptation to equine species as it had only been mentioned in studies performed on horses in China, Colombia, the Czech Republic, Algeria, and the USA [8$12,14]$. Apart from a single case in a NHP [25], we identified this genotype in donkeys in this study. Genotypes BEB4 and BEB6 are mostly found in cattle, humans, and other animals $[3,26]$. BEB4 has previously been identified in humans, pigs, and NHPs $[18,19,27]$, whilst BEB6 has been found in humans, NHPs, alpacas, deer, goats, sheep, takin, yak, cats, horses, mice, birds, and wastewater $[3,26]$. Initially, both BEB4 and BEB6 were discovered in donkeys indicating that their reservoir hosts may be more expansive than predicted. Genotype NIA1 was first identified in an AIDS patient from Nigeria [28], and in an HIV-infected patient from Congo [29]. Until now, none of the studies have reported the presence of genotype NIA1 in any animal species other than humans. This study is the first report on the identification of genotype NIA1 in donkeys confirming potential zoonosis. 
It is known that genotypes belonging to groups 1 and 2 represent a large number of hosts, including humans, and may cause most of the zoonotic E. bieneusi infections [3]. Here, all genotypes were clustered into either group 1 (horse1, D, NIA1, NCD-2, and XJD2) or group 2 (BEB6, BEB4, and XJD1) (Fig. 1), implying that E. bieneusi-infected donkeys could be a potential threat to humans.

\section{Conclusions}

This study first identifies E. bieneusi in donkeys in Xinjiang, China. We found a relatively low prevalence of E. bieneusi in both farmed and free-ranging donkeys. However, a high level of genetic variation was identified amongst the infected donkeys, with zoonotic genotypes D, BEB6, BEB4, and NIA1. ITS sequencing-based phylogenetic analysis revealed that all the $E$. bieneusi isolates came from donkeys belonging to Groups 1 or 2 . These data indicate the possibility of donkey-to-human transmission of this pathogenic parasite.

\section{Methods \\ Sample collection}

Six hundred and eighty fecal samples (approximately 50 g each) were gathered from 178 free-ranging donkeys in five countrysides, and 502 farmed donkeys from 18 farms in 13 cities of Xinjiang, China, between May 2016 to December 2018 (Table 1). A sterile disposable latex glove was worn while collecting the fecal samples, immediately post-defecation and placed in labeled sterile bags. Each animal was identified based on neck ropes, ear tags, and body features such as color and size to avoid sample duplication. The collected samples accounted for 10 to $30 \%$ of adults or young donkeys on each farm and all free-ranging donkeys in the countryside. The samples were transported under ice-cold conditions and stored at $4{ }^{\circ} \mathrm{C}$ until further use. The adult and young donkeys were $\geq 1$ year and $<1$ year old, respectively. No clinical symptom was observed in any ani$\mathrm{mal}$ at the time of sampling.

\section{DNA extraction}

A suspension was created by mixing the feces sample (approximately $10 \mathrm{~g}$ ) with $30 \mathrm{~mL}$ of distilled water, filtered, and centrifuged for $5 \mathrm{~min}$ at $3000 \mathrm{~g}$. We used the E.Z.N.A. stool DNA kit to extract the genomic DNA from $200 \mathrm{mg}$ of the fecal samples (pellets), following the manufacturer's protocol. The isolated DNA $(200 \mu \mathrm{L})$ from each sample was preserved at $-20^{\circ} \mathrm{C}$ before PCR analysis.

\section{PCR}

A $390 \mathrm{bp}$ region of the rRNA gene was amplified using the $2 \times$ EasyTaq PCR SuperMix to examine the prevalence of $E$. bieneusi. Primary and secondary PCR amplifications were performed using two pairs of primers, EBITS1 and EBITS2.4, and EBITS3 and EBITS4, resulting in $390 \mathrm{bp}$ and $\sim 435$ bp fragments, respectively [30]. Their respective parameters were: 30 cycles of $94{ }^{\circ} \mathrm{C}$ for $30 \mathrm{~s}, 55^{\circ} \mathrm{C}$ for $30 \mathrm{~s}$, and $72{ }^{\circ} \mathrm{C}$ for $40 \mathrm{~s}$; and 35 cycles of $94{ }^{\circ} \mathrm{C}$ for $30 \mathrm{~s}, 57^{\circ} \mathrm{C}$ for $30 \mathrm{~s}$, and $72^{\circ} \mathrm{C}$ for $40 \mathrm{~s}$; with a final extension step of $72^{\circ} \mathrm{C}$ for $10 \mathrm{~min}$ [30]. Distilled water was used as the negative control and DNA from dairy cattle-derived genotype I was used as the positive control.

\section{Sequencing}

GENEWIZ (Suzhou, China) was used to sequence the $E$. bieneusi-positive secondary PCR products. Bi-directional sequencing and the sequencing of PCR products was done to confirm sequence accuracy when required for DNA preparations. The E. bieneusi genotypes were detected by sequence alignment using ClustalX v1.83. If the identified genotypes of $E$. bieneusi were similar to the ones in the GenBank database, then they were given the first published name; however, if DNA sequencing confirmed any single nucleotide substitutions/deletions/ insertion in the ITS sequences of minimum two PCR products, then they were labeled as new genotypes. All samples were given a genotype identity by adding Arabic numbers after the abbreviation XJD (Xinjiang Donkey) based on the order of appearance. All genotypes were labeled using the established nomenclature system [7].

\section{Phylogenetic analysis}

A neighbor-joining phylogenetic tree was constructed using Mega 7.0 based on the Kimura-2-parameter model to verify the genogroup designation and to evaluate the genetic relationships between the novel identified ITS genotypes of $E$. bieneusi with known sequences. Bootstrap analysis with 1000 replicates was used to assess the reliability of the phylogenetic trees.

\section{Abbreviations}

PCR: Polymerase chain reaction; ITS: Internal transcribed spacer; HIV: Human immunodeficiency virus; NHP: Non-human primates

\section{Acknowledgements}

Not applicable.

\section{Authors' contributions}

$M Q$ and $W Z$ conceived the study and contributed to the design. $A Z, Y Z$, WW, BJ, JX and DT contributed to acquisition of samples and performed experiments. AZ and WZ contributed to data analysis. WZ and MQ contributed to writing the manuscript. MQ and AZ obtained funding. All authors approved of the final version to be published and agree to be accountable for all aspects of the manuscript.

\section{Funding}

This study was partly supported by the National Natural Science Foundation of China (31660712 and 31860699), the Program for Young and Middle-aged Leading Science, Technology and Innovation of Xinjiang Production \& Construction Group (2018CB034). The sponsors played no roles in the study design, or in the collection, analysis, or interpretation of the data, in writing the report, or in the decision to submit the article for publication. 


\section{Availability of data and materials}

All data generated and/or analyzed during this study are included in this published manuscript. The nucleotide sequences identified in this study were deposited under accession numbers MN174113 to MN174120 in the GenBank database.

\section{Ethics approval and consent to participate}

The research protocol was reviewed and approved by the Research Ethics Committee and the Animal Ethical Committee of Tarim University. Before beginning work on the present study, we contacted the donkey farm owners and written informed consent was obtained from the owners for the participation of their animals in this study. During collecting fecal specimens, these animals were not disturbed.

\section{Consent for publication}

Not applicable.

\section{Competing interests}

The authors declare that they have no competing interests.

Received: 17 October 2019 Accepted: 3 June 2020

Published online: 15 June 2020

\section{References}

1. Fayer R, Santin-Duran M. Epidemiology of microsporidia in human infections. In: Weiss LM, Becnel JJ, editors. Microsporidia: pathogens of opportunity. 1st ed. Chichester: Wiley; 2014. https://doi.org/10.1002/ 9781118395264.ch3.

2. Matos O, Lobo ML, Xiao L. Epidemiology of Enterocytozoon bieneusi infection in humans. J Parasitol Res. 2012;2012:981424. https://doi.org/10. $1155 / 2012 / 981424$

3. Li W, Feng Y, Santin M. Host specificity of Enterocytozoon bieneusi and public health implication. Trends Parasitol. 2019;35:436-51. https://doi.org/ 10.1016/j.pt.2019.04.004.

4. Santín M, Fayer R. Microsporidiosis: Enterocytozoon bieneusi in domesticated and wild animals. Res Vet Sci. 2011;90:363-71. https://doi.org/10.1016/j.rvsc. 2010.07.014

5. Decraene V, Lebbad M, Botero-Kleiven S, Gustavsson AM, Löfdahl M. First reported foodborne outbreak associated with microsporidia, Sweden, October 2009. Epidemiol Infect. 2012;140:519-27. https://doi.org/10.1017/ S095026881100077X.

6. Li N, Xiao L, Wang L, Zhao S, Zhao X, Duan L, et al. Molecular surveillance of Cryptosporidium spp., Giardia duodenalis, and Enterocytozoon bieneusi by genotyping and subtyping parasites in wastewater. PLoS Negl Trop Dis. 2012;6:e1809. https://doi.org/10.1371/journal.pntd.0001809.

7. Santín M, Fayer R. Enterocytozoon bieneusi genotype nomenclature based on the internal transcribed spacer sequence: a consensus. J Eukaryot Microbiol. 2009;56:34-8.

8. Laatamna AE, Wagnerová $P$, Sak B, Květoňová $D$, Xiao L, Rost M, et al. Microsporidia and Cryptosporidium in horses and donkeys in Algeria: detection of a novel Cryptosporidium hominis subtype family (Ik) in a horse. Vet Parasitol. 2015;208:135-42. https://doi.org/10.1016/j.vetpar.2015.01.007.

9. Santín $M$, Vecino JA, Fayer R. A zoonotic genotype of Enterocytozoon bieneusi in horses. J Parasitol. 2010;96:157-61. https://doi.org/10.1645/GE-2184.1.

10. Deng L, Li W, Zhong Z, Gong C, Liu X, Huang X, et al. Molecular characterization and multilocus genotypes of Enterocytozoon bieneusi among horses in southwestern China. Parasit Vectors. 2016;9:561. https:// doi.org/10.1186/s13071-016-1844-3.

11. Wagnerová $P$, Sak $B$, Květoňová $D$, Buňatová $Z$, Civišová $H$, Maršálek $M$, et al. Enterocytozoon bieneusi and Encephalitozoon cuniculi in horses kept under different management systems in the Czech Republic. Vet Parasitol. 2012; 190:573-7. https://doi.org/10.1016/j.vetpar.2012.07.013.

12. Qi M, Wang R, Wang H, Jian F, Li J, Zhao J, et al. Enterocytozoon bieneusi genotypes in grazing horses in China and their zoonotic transmission potential. J Eukaryot Microbiol. 2016;63:591-7. https://doi.org/10.1111/jeu. 12308.

13. Yue DM, Ma JG, Li FC, Hou JL, Zheng WB, Zhao Q, et al. Occurrence of Enterocytozoon bieneusi in donkeys (Equus asinus) in China: a public health concern. Front Microbiol. 2017:8:565. https://doi.org/10.3389/fmicb.2017. 00565.
14. Li J, Qi M, Chang Y, Wang R, Li T, Dong H, et al. Molecular characterization of Cryptosporidium spp., Giardia duodenalis, and Enterocytozoon bieneusi in captive wildlife at Zhengzhou zoo, China. J Eukaryot Microbiol. 2015;62:8339. https://doi.org/10.1111/jeu.12269.

15. Zhao W, Wang J, Ren G, Yang Z, Yang F, Zhang W, et al. Molecular characterizations of Cryptosporidium spp. and Enterocytozoon bieneusi in brown rats (Rattus norvegicus) from Heilongjiang Province, China. Parasit Vectors. 2018;11:313. https://doi.org/10.1186/s13071-018-2892-7.

16. Wang H, Liu Q, Jiang X, Zhang Y, Zhao A, Cui Z, et al. Dominance of zoonotic genotype $D$ of Enterocytozoon bieneusi in bamboo rats (Rhizomys sinensis). Infect Genet Evol. 2019;73:113-8. https://doi.org/10.1016/j.meegid. 2019.04.025

17. Zhao W, Zhang W, Yang F, Zhang L, Wang R, Cao J, et al. Enterocytozoon bieneusi in dairy cattle in the northeast of China: genetic diversity of ITS gene and evaluation of zoonotic transmission potential. J Eukaryot Microbiol. 2015;62:553-60. https://doi.org/10.1111/jeu.12210.

18. Zhang X, Wang Z, Su Y, Liang X, Sun X, Peng S, et al. Identification and genotyping of Enterocytozoon bieneusi in China. J Clin Microbiol. 2011;49: 2006-8. https://doi.org/10.1128/JCM.00372-11.

19. Tang C, Cai M, Wang L, Guo Y, Li N, Feng Y, et al. Genetic diversity within dominant Enterocytozoon bieneusi genotypes in pre-weaned calves. Parasit Vectors. 2018;11:170. https://doi.org/10.1186/s13071-018-2768-x.

20. Yu F, Wu Y, Li T, Cao J, Wang J, Hu S, et al. High prevalence of Enterocytozoon bieneusi zoonotic genotype D in captive golden snub-nosed monkey (Rhinopithecus roxellanae) in zoos in China. BMC Vet Res. 2017;13: 158. https://doi.org/10.1186/s12917-017-1084-6.

21. Qi M, Jing B, Jian F, Wang $R$, Zhang $S$, Wang $H$, et al. Dominance of Enterocytozoon bieneusi genotype $J$ in dairy calves in Xinjiang, Northwest China. Parasitol Int. 2017;66:960-3. https://doi.org/10.1016/j.parint.11.

22. Ye J, Ji Y, Xu J, Ma K, Yang X. Zoonotic Enterocytozoon bieneusi in raw wastewater in Zhengzhou, China. Folia Parasitol. 2017;18:64. https://doi.org/ 10.14411/fp.2017.002.

23. Zhang Y, Koehler AV, Wang T, Haydon SR, Gasser RB. First detection and genetic characterisation of Enterocytozoon bieneusi in wild deer in Melbourne's water catchments in Australia. Parasit Vectors. 2018;11:2. https://doi.org/10.1186/s13071-017-2577-7.

24. Pirestani M, Sadraei J, Forouzandeh M. Molecular characterization and genotyping of human related microsporidia in free-ranging and captive pigeons of Tehran, Iran. Infect Genet Evol. 2013;20:495-9.

25. Zhong Z, Li W, Deng L, Song Y, Wu K, Tian Y, et al. Multilocus genotyping of Enterocytozoon bieneusi derived from nonhuman primates in Southwest China. PLoS One. 2017;12:e0176926. https://doi.org/10.1371/journal.pone. 0176926.

26. Zhao W, Wang J, Yang Z, Liu A. Dominance of the Enterocytozoon bieneusi genotype BEB6 in red deer (Cervus elaphus) and Siberian roe deer (Capreolus pygargus) in China and a brief literature review. Parasite. 2017;24:54. https:// doi.org/10.1051/parasite/2017056.

27. Karim MR, Dong H, Li T, Yu F, Li D, Zhang L, et al. Predomination and new genotypes of Enterocytozoon bieneusi in captive nonhuman primates in zoos in China: high genetic diversity and zoonotic significance. PLoS One. 2015;10:e0117991. https://doi.org/10.1371/journal.pone.0117991.

28. Espern A, Morio F, Miegeville M, Illa H, Abdoulaye M, Meyssonnier V, et al. Molecular study of microsporidiosis due to Enterocytozoon bieneusi and Encephalitozoon intestinalis among human immunodeficiency virus-infected patients from two geographical areas: Niamey, Niger, and Hanoi, Vietnam. J Clin Microbiol. 2007;45:2999-3002.

29. Wumba R, Longo-Mbenza B, Mandina M, Odio WT, Biligui S, Sala J, et al. Intestinal parasites infections in hospitalized AIDS patients in Kinshasa, Democratic Republic of Congo. Parasite. 2010;17:321-8.

30. Buckholt MA, Lee JH, Tzipori S. Prevalence of Enterocytozoon bieneusi in swine: an 18-month survey at a slaughterhouse in Massachusetts. Appl Environ Microbiol. 2002;68:2595-9. https://doi.org/10.1128/AEM.68.5.25952599.2002.

\section{Publisher's Note}

Springer Nature remains neutral with regard to jurisdictional claims in published maps and institutional affiliations. 